\title{
Cirrhotic cardiomyopathy: we already know. What comes next?
}

\author{
Luís Otávio Mocarzel $^{1}$, Jessica Bicca ${ }^{1}$, Luiza Jarske ${ }^{1}$, Thamires Oliveira ${ }^{1}$, Pedro Lanzieri ${ }^{1}$, and Ronaldo Altenburg Gismondi ${ }^{1}$ \\ ${ }^{1}$ Department of Clinical Medicine, Antonio Pedro University Hospital, Fluminense Federal University, Rio de Janeiro, Brazil
}

\begin{abstract}
Cirrhotic Cardiomyopathy is a recently recognized condition consisting of myocardial dysfunction manifested in cirrhotic patients in the absence of previous cardiac disease. The underlying pathogenetic mechanisms includes a "miocardiotoxic" environment that leads to a blunted heart response to stress, whether physical or pharmacological. This condition is now better understood and can be diagnosed supported clinical, by laboratory electrocardiography and echocardiography criteria. Clinically, systolic incompetence is most evident when cirrhotic patients are placed under stress, whether physical or pharmacological. Despite of being well understood, there is a lack of knowledge to a proper practical application. Recent case reports have suggested that CCM must be treated early because it may be an triggering factor for cirrhosis complications. Studies are needed to identify potential treatments that alter the natural history of heart disease in cirrhotic patients, especially in the phases in which the patients are asymptomatic.
\end{abstract}

\section{Introduction}

Cirrhotic cardiomyopathy (CCM) is a cardiac dysfunction characterized by suboptimal contractile response to stress and impaired diastolic function in the absence of previous cardiac disease [1-3]. Nowadays, research has proposed that cirrhotic patients have an intrinsic cardiac dysfunction that may influence in the development of renal failure in advanced liver disease [4].

Since 2002, the findings about circulatory dysfunction in liver disease have evolved through diagnostic methods, emphasizing the importance of identification of CCM in clinical practice [5]. However, there's still a gap of knowledge regarding management of this condition [6]. The presence of myocardial dysfunction in cirrhosis increases the risk of hepatorrenal syndrome (HRS) and adverse cardiac events and it is related to higher mortality [3].

We reviewed the available literature in PubMed regarding the markers for cirrhosis complications and the interactions of the heart and the liver. The purpose of this review is to discuss the need to early track and treat CCM, since it may be a triggering factor for cirrhosis complications, thus bringing new insights to clinical practice.

\section{Definition and prevalence}

CCM describes cardiac chronic dysfunction in cirrhotic patients despite no previous structural heart disease [1]. It is defined by one or more of the following: structural abnormalities in cardiac chambers; diastolic dysfunction; normal or augmented systolic function at rest but poor contractile response to stress; and electrophysiological changes [2]. These abnormalities may be observed in up to $50 \%$ of cirrhotic patients [3].

\section{Physiopathology}

Cirrhosis physiopathology is known by overproduction of nitric oxide (NO) in splanchnic vessels and consequent systemic vasodilatation. This mechanism triggers rennin-angiotensin- aldosterone system (RAAS) and sympathetic nervous system activation and rises serum vasopressin level, leading to a hyperdinamic state that may repercute from cellular to clinical settings.

These patients have an increased serum carbon monoxide (CO) secondary to sympathetic hyperactivity and inflammatory cytokines. There is a rise in CO, associated with high NO levels, accumulation of endocannabinoids and other vasodilators. These substances increases cyclic guanosine monophosphate (cGMP) leves, which inhibits cyclic adenosine monophosphate (cAMP) in cardiomyocites, responsible for calcium influx and greater contractile force (Figure 1,2) [7]. Myocardial cells analysis show a reduction in plasmatic membrane fluidity due to cholesterol deposition, which difficult the interaction of substances such as beta-adrenergic receptors and G-proteins $[7,8]$. The ventricular myocite may also have a decrease in potassium channels function, which leads to a suboptimal cardiac relaxation, and consequent diastolic dysfunction $[8,9]$.

The persistent beta-adrenergic stimuli induces down-regulation and reduces cardiac receptors density, modifying signaling cascades responsible for calcium channel activation [9]; the outcome is an impaired contractile function [5]. Clinical repercussion of this pathway is a blunted myocardial function and an increase in cardiac biomarkers [7].

Identification of CCM relies on subtle clinical features that may be unmasked as the disease progresses, or can become clearly evident in specific clinical scenarios of strain, and becomes a challenge regarding whether and when to treat.

Correspondence to: Pedro Gemal Lanzieri, Department of Clinical Medicine, Antonio Pedro University Hospital, Fluminense Federal University, Rio de Janeiro, Brazil, Tel: +55-21-26299210; Email: pedrogemal@id.uff.br

Key words: liver cirrhosis, heart failure, cardiomyopathy

Received: February 15, 2017; Accepted: March 01, 2017; Published: March 03, 2017 


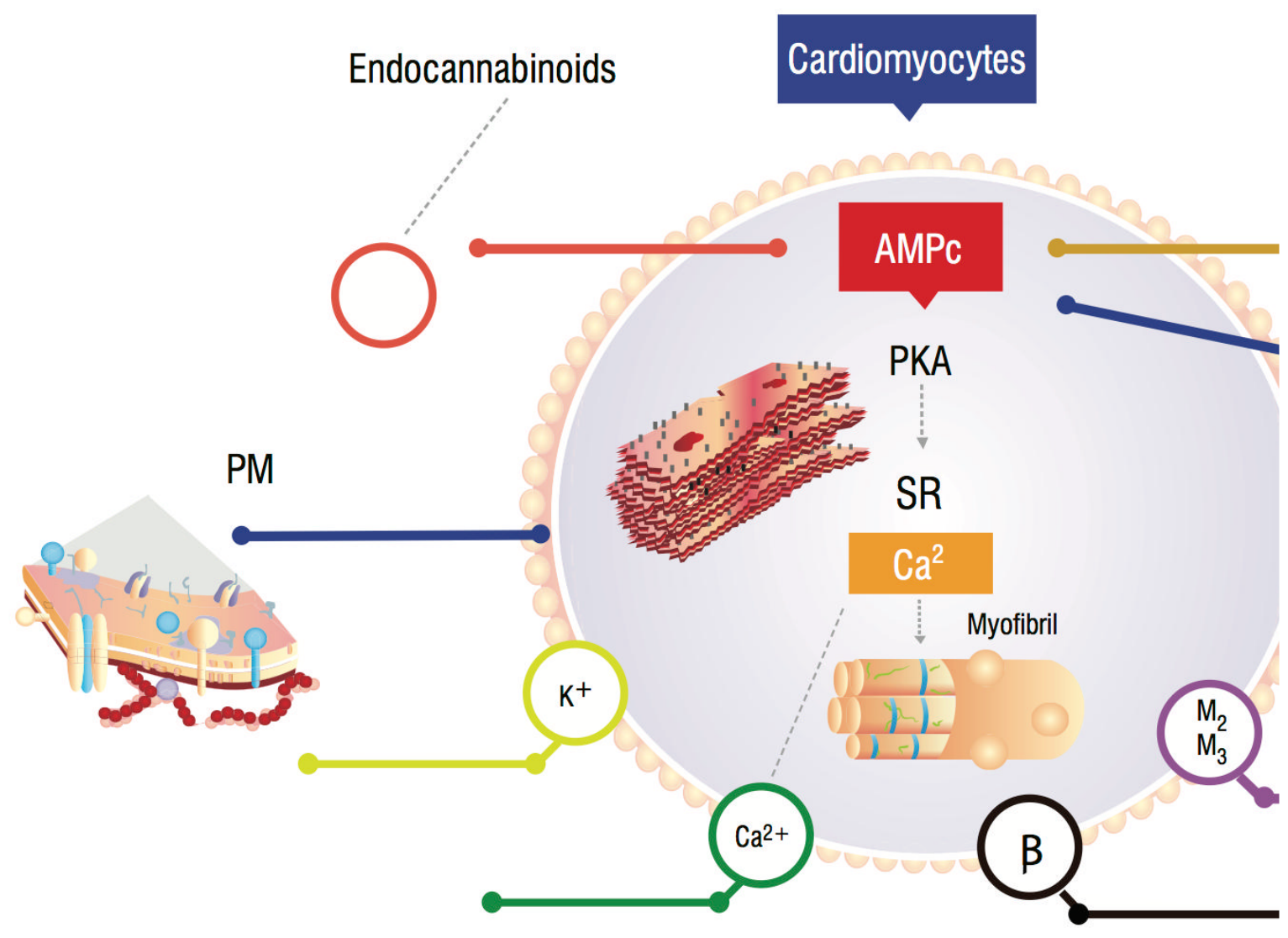

Figure 1. Physiopathological model of cardiomyocyte showing main signaling pathways that leads to a blunded myocardial response to stress. PM: Plasmatic membrane; CO: Carbon oxide; NO: Nitric oxide; TNF-a: Tumor necrosis factor alfa; SR: Sarcoplasmatic reticullum; AMPc: Cyclic adenosine monophosphate; PKA: Protein kinase A.
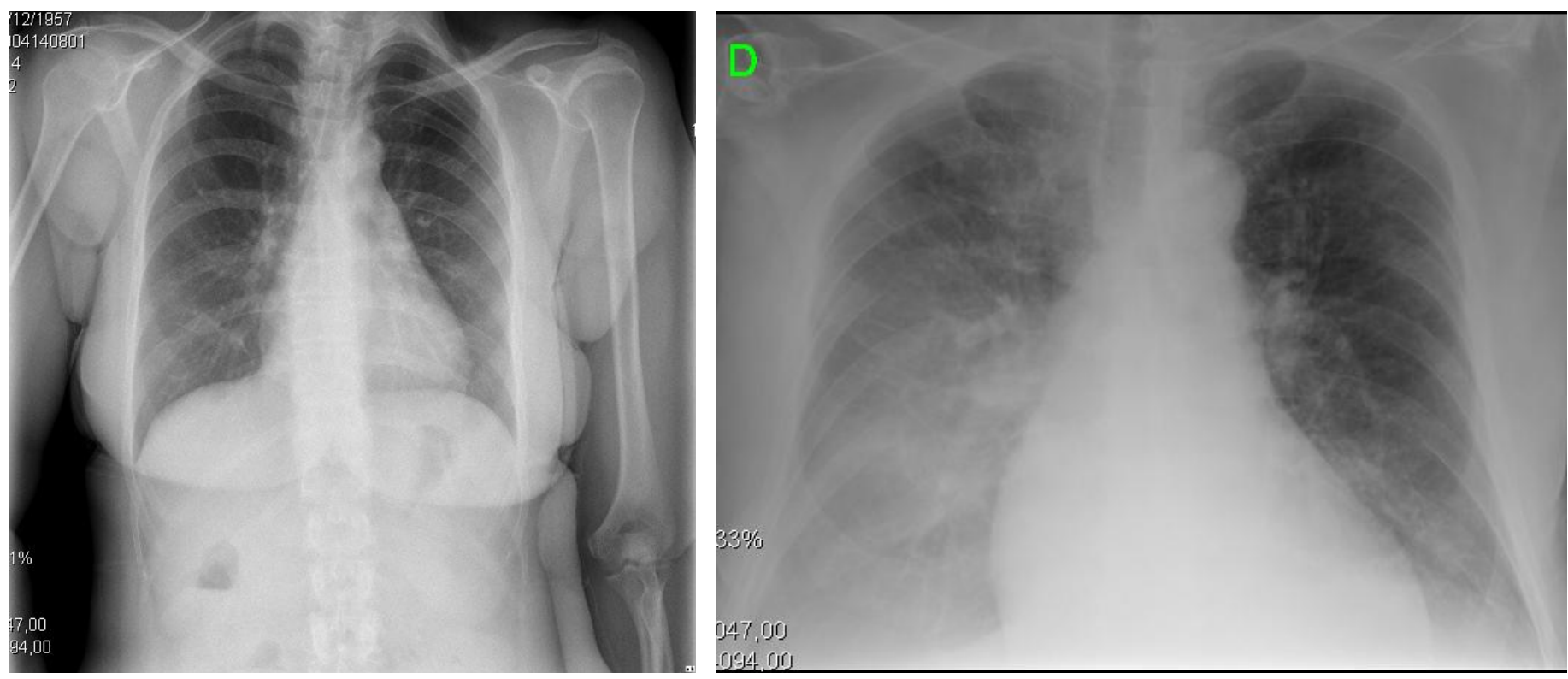

Figure 2. Clinical and radiologic presentations of different evolutionary stages of cirrhotic cardiomyopathy. A. Left image shows small pleural effusion and CTI of 0.50 . B. Right image depicts a severe pulmonary congestion and CTI of 0.67 . 


\section{Natural history and diagnosis}

Diagnostic criteria are summarized in table 1 and relies on clinical, imaging and biomarkers.

\section{Clinical features}

Clinical features of CCM may occur with patients with an apparently normal cardiac function, since chronic adaptation of the cardiomyocyte to the cirrhotic environment leads to all those cellular modifications [7].

Cirrhotic patients evolve with sodium and fluid retention, peripheral vasodilatation, sympathetic hyperactivity and accumulation of "miocardiotoxic" substances, demanding an adaptive process of cardiomyocyte. This continuum balance can be broken and unmask a latent heart failure in stress situations, such as liver transplant, transjugular intrahepatic portosystemic shunt or infections $[8,9]$.

Although cirrhotic cardiomyopathy tends to be a chronic condition, studies reveal that clinical signs of myocardial dysfunction can reverse in up to 15 months after successful liver transplant, as well as the QT prolongation [10].

\section{Laboratory findings}

Since cardiac dysfunction may be reflected by inflammatory biomarkers, novel cardiac and pro-inflammatory markers are being recently studied and correlated to the development of complications leading to increased mortality [11].

Recent studies are describing correlation between levels of prohormone of ANP (proANP) and B-type natriuretic peptide (BNP), copeptin and high-sensitivity troponin $\mathrm{T}$ (hs-TnT), as well as soluble urokinase-type plasminogen activator receptor (suPAR) and highsensitive C-reactive protein (hs-CRP) and cardiovascular complications in cirrhosis. However, its clinical usage is still being evaluated [12,13].

Natriuretic peptide are secreted by the cardiomyocytes in response to volume or pressure overload, or myocardial ischemia, to compensate RAAS effect in sodium and water balance $[14,15]$. BNP may be one of the most available biomarkers which allows an early recognition of cardiac involvement $[16,17]$. It's serum concentration is higher the more severe the liver and heart diseases [18].

Promising studies are stating that high levels of adrenomedullin are also seen in cirrhosis with or without clinical cardiac involvement;

Table 1. Clinical and complimentary criteria for the diagnosis of CCM. Adapted from from reference 29 with permission.

\begin{tabular}{|l|}
\hline Clinical features \\
\hline No cardiopulmonary symptoms at rest \\
Low functional cardiac reserve \\
\hline Hiperadrenergic state with sodium and fluid retention \\
Biomarkers (troponin I, pro-BNP and BNP, others) \\
Eletrocardiography \\
Electrophysiological abnormalities (QT prolongation and others) \\
Echocardiographic criteria \\
\hline Diastolic evaluation \\
\hline E/A ratio $<1.0$ (age corrected) \\
\hline Prolonged isovolumic relaxation time $(>80 \mathrm{msec})$ \\
\hline Prolonged deceleration time $(>200 \mathrm{msec})$ \\
\hline Left ventricular hypertrophy \\
Systolic evaluation \\
\hline Ejection fraction at rest $<55 \%$ \\
\hline Contractile deficit in stress \\
\hline
\end{tabular}

this substance is released in blood flow to act as a vasodilator and aims to reduce afterload [18]. Galectin-3, the most recent studied biomarker elevated in cirrhotic patients, has been associated with myocardial fibrosis and may correlates with severity of cirrhosis. This marker is proving to be more sensitive than BNP in the evaluation of diastolic dysfunction, though is less specific [16].

\section{Electrocardiogram}

Electrocardiographic (ECG) abnormalities can be early noticed in a asymptomatic CCM patient and may correlate with ventricular arrhythmias and sudden death [3]. The most common ECG finding is QT interval prolongation, which may be observed in approximately $30-40 \%$ of cirrhotic patients, mainly Child-Pugh B or C, regardless cirrhosis etiology $[19,16]$.

This ECG pattern may be secondary to cardiomyocytes repolarization abnormalities in potassium channels and reduction in the concentration of these channels in the plasma membrane, in response to minor modifications to the portal pressure [20]. Ionic channels dysfunctions thereby causes myocardial cells action potential prolongation, resulting in longer ventricular systole and QT [21-23]. Longer contraction time implies impaired relaxation, causing diastolic dysfunction $[24,25]$.

Electromechanical uncoupling is described as a dyssynchrony between electrical stimulus and systolic mechanical response of the heart, taking to progressive loss of myocardial function and congestive heart failure in these patients1 [7]. The chronotropic incompetence is characterized by inability of sinus node to respond to physiological or pharmacological stimuli [23], an determinant fact in CCM physiopathology. These changes are also secondary to portal hypertension, autonomic dysfunction, hyperdynamic circulation and the presence of the pro-inflammatory factors affecting the patient's myocardium $[9,18]$.

\section{Echocardiogram}

Echocardiogram provides relevant data about the cardiac function and is used as diagnostic criteria for cirrhotic cardiomyopathy [26].

The main findings in these patients are end-diastolic left ventricle (LV) enlargement, reduction of peak velocity and systolic deformity rate; increased end-diastolic left ventricular pressure, without appropriate LVEF expansion. Left atrium (LA) enlargement for more than $34 \mathrm{~mL} /$ $\mathrm{m}^{2}$ may suggests chronic diastolic dysfunction and, when associated with reduction of LV mass, may suggest worse prognosis [4,27]. LA evaluation can be made through two dimension echocardiography and speckle tracking [28].

Common findings observed in the cardiac doppler echocardiography indicate myocardial relaxation impaired in cirrhotic patients: decrease of $\mathrm{E} / \mathrm{A}$ ratio $(<1)$, and prolonged $\mathrm{E}$ wave deceleration time (DT) and isovolumetric relaxation time (IVRT) $>80 \mathrm{~ms}$ [27-30]. The more severe is the diastolic dysfunction, shorter is the IVRT and mitral DT, and higher is the E/A velocity ratio, characterizing advanced diastolic dysfunction, increased LA pressure, and worse functional class.

Recent studies with global longitudinal strain (GLS) evaluation shows that this may be a helpful method for the early detection of cardiac dysfunction in the cirrhotic patient, mainly the systolic dysfunction at rest $[3,28]$.

E/A ratio may be a reliable parameter in the diagnosis of diastolic dysfunction in cirrhotic patients, even with values influenced by the degree of hydration of the patient, although it may vary due to ascites 
and a volume overload [4]. Meanwhile, the isovolumetric relaxation time, although highly sensitive, does not represent the LV filling and varies as the heart rate changes [16].

A mild systolic dysfunction and a diastolic dysfunction can be presented independently of the degree of cirrhosis. However, the diastolic dysfunction is found most commonly in cirrhotic patients and generally precedes the former [4].

\section{Discussion}

This review addresses an increasing identification and understanding of physiopathology on CCM, it's correlation with poor prognosis in cirrhotic patients and arises the concern about therapeutic approach.

This article summarized the diagnostic criteria (Table 1) already established in the medical literature, with emphasis on the new biomarkers that have been studied for diagnostic complementation. Studies have shown that myocardial dysfunction exists in various stages of liver cirrhosis. It has been described that if the patient has high BNP and ventricular dysfunction on the echocardiogram, treating the kidney failure is very difficult; On the other hand, early intervention in patients who do not yet have established heart disease is an area that has not yet been explored.

The signs and symptoms of liver failure tend to be late, with subtle clinical and laboratory manifestations, sometimes difficult to interpret. The specialist, or even the general practitioner, may face a patient with dyspnea complaint, without pathological jugular swelling, normal initial cardiology exams, but with high BNP, which may represent a case of CCM.

In contrast to other studies, our group proposes an advance in this field, since the understanding of heart disease concomitant with cirrhosis may allow an early therapeutic intervention of complications attributable to undiagnosed myocardial failure. Liver transplantation and TIPS are special clinical settings that may deflagrate heart failure in these patients. As patients survive longer after these procedures, clinicians would be faced with worsening cardiac parameters [21].

We believe that early therapeutic intervention in CCM is promising, when it is manifested by complications such as refractory HRS, before presenting clear pulmonary congestion attributable to myocardial failure. Literature supports that situations of physical or pharmacological stress, or infections, associated to latent cirrhotic cardiomyopathy may result in HRS, that may be refractory to conventional treatments and associated with worse prognosis if systolic dysfunction is present [9].

Our experience suggests that presentation of HRS is a clue to initiate the treatment of the myocardial component. This progression to HRS is understood since existence of CCM is a trigger and perpetuating factor of HRS, so if CCM is approached early in this context, HRS can have a better prognosis [21].

Our group has pioneered the treatment of patients with a clinical diagnosis of CCM in progression to SHR refractory to conventional treatments, in which the use of dobutamine as a rescue therapy for renal function was successful. The goal is to apply this theoretical knowledge anticipating this complication in end-stage liver disease patients [6].

Despite of being well understood, there is a lack of knowledge to a proper practical application. A high degree of clinical suspicion is required to recognize CCM in the asymptomatic patient with no apparent functional limitations, but with subclinical cellular and structural heart disease.

Studies are needed to identify potential treatments that alter the natural history of heart disease in cirrhotic patients, especially in the phases in which the patients are asymptomatic. This represents an area of knowledge that has not yet been explored, specially regarding future therapeutic options.

\section{Conflicts of interest}

The authors declared that there are no conflicts of interests regarding the publication of this paper.

\section{Financial support}

None.

References

1. Sampaio F, Pimenta J (2016) Left ventricular function assessment in cirrhosis: Current methods and future directions. World J Gastroenterol 22: 112-125. [Crossref]

2. Shin WJ, Song JG, Jun IG, Moon YJ, Kwon HM, et al. (2016) Effect of ventriculoarterial coupling on transplant outcomes in cirrhotics: Analysis of pressure-volume curve relations. $J$ Hepatol. [Crossref]

3. Carvalheiro F, Rodrigues C, Adrego T, Viana J, Vieira H, et al. (2016) Diastolic Dysfunction in Liver Cirrhosis: Prognostic Predictor in Liver Transplantation? Transplant Proc 48: 128-31. [Crossref]

4. Merli M, Torromeo C, Giusto M, Iacovone G, Riggio O, et al. (2016) Survival at 2 years among liver cirrhotic patients is influenced by left atrial volume and left ventricular mass. Liver Int. [Crossref]

5. Møller S, Henriksen JH (2002) Cirrhotic cardiomyopathy: a pathophysiological review of circulatory dysfunction in liver disease. Heart 87: 9-15. [Crossref]

6. Mocarzel LO, Bicca J, Jarske L, Oliveira T, Lanzieri P, et al. (2016) Cirrhotic Cardiomyopathy: Another Case of a Successful Approach to Treatment of Hepatorenal Syndrome. Case Rep Gastroenterol 10: 531-7. [Crossref]

7. Timoh T, Protano MA, Wagman G, Bloom M, Vittorio TJ (2011) A perspective on cirrhotic cardiomyopathy. Transplant Proc 43: 1649-1653. [Crossref]

8. Ma Z, Lee SS (1996) Cirrhotic cardiomyopathy: getting to the heart of the matter. Hepatology 24: 451-459. [Crossref]

9. Zardi EM, Abbate A, Zardi DM, Dobrina A, Margiotta D, et al. (2010) Cirrhotic cardiomyopathy. J Am Coll Cardiol 56: 539-549. [Crossref]

10. Myers RP, Lee SS (2000) Cirrhotic cardiomyopathy and liver transplantation. Liver Transpl 6: S44-52. [Crossref]

11. Wiese S, Mortensen C, Gøtze JP, Christensen E, Andersen O, et al. (2014) Cardiac and proinflammatory markers predict prognosis in cirrhosis. Liver Int 34: e19-30. [Crossref]

12. Fattouh AM, El-Shabrawi MH, Mahmoud EH, Ahmed WO (2016) Evaluation of cardiac functions of cirrhotic children using serum brain natriuretic peptide and tissue Doppler imaging. Ann Pediatr Cardiol 9: 22-8. [Crossref]

13. Rahman S, Mallett S V (2015) Cirrhotic cardiomyopathy?: Implications for the perioperative management of liver transplant patients 7: 507-20. [Crossref]

14. Farr M, Schulze PC (2015) Recent Advances in the Diagnosis and Management of Cirrhosis-Associated Cardiomyopathy in Liver Transplant Candidates: Advanced Echo Imaging, Cardiac Biomarkers, and Advanced Heart Failure Therapie. Clin Med Insights Cardiol 8: 67-74. [Crossref]

15. Licata A, Corrao S, Petta S, Genco C, Cardillo M, et al. (2013) NT pro BNP plasma level and atrial volume are linked to the severity of liver cirrhosis. PLoS One 8: e68364. [Crossref]

16. Abbas WA, Kasem Ahmed SM, Abdel Aal AM, Mahmoud AA, Abdelmalek MO, Mekky MA, et al. Galactin-3 and brain natriuretic peptide versus conventional echocardiography in the early detection of cirrhotic cardiomyopathy. Turk $J$ Gastroenterol 27: 367-74. [Crossref]

17. Wong F, Siu S, Liu P, Blendis LM (2001) Brain natriuretic peptide: is it a predictor of cardiomyopathy in cirrhosis? Clin Sci (Lond) 101: 621-628. [Crossref]

18. Wong F (2009) Cirrhotic cardiomyopathy. Hepatol Int 3: 294-304. [Crossref] 
19. Mimidis K, Papadopoulos V, Thomopoulos K, Tziakas D, Ritis K, et al. (2003) Prolongation of the QTc interval in patients with cirrhosis. Ann Gastroenterol 16 : $155-8$

20. Mozos I (2015) Arrhythmia risk in liver cirrhosis. World J Hepatol 7: 662-672. [Crossref]

21. Mocarzel LO, Bicca J, Jarske L, Oliveira T, Lanzieri P, et al. (2016) Cirrhotic Cardiomyopathy: Another Case of a Successful Approach to Treatment of Hepatorenal Syndrome. Case Rep Gastroenterol 10: 531-537. [Crossref]

22. Batchvarov V, Camm AJ (2014) QT dispersion: Measurement and interpretation.

23. Páll A, Czifra A, Vitális Z, Papp M, Paragh G, et al. (2014) Pathophysiological and clinical approach to cirrhotic cardiomyopathy. J Gastrointestin Liver Dis 23: 301-310. [Crossref]

24. Castro Hevia J, Antzelevitch C, Tornés Bárzaga F, Dorantes Sánchez M, Dorticós Balea F, et al. (2006) Tpeak-Tend and Tpeak-Tend dispersion as risk factors for ventricular tachycardia/ventricular fibrillation in patients with the Brugada syndrome. $\mathrm{J} \mathrm{Am} \mathrm{Coll}$ Cardiol 47(9): 1828-34. [Crossref]
25. Demir C, Demir M (2013) Evaluation of Tp-e interval and Tp-e/QT ratio in patients with chronic hepatitis B. Prague Med Rep 114: 239-245. [Crossref]

26. Jewell DP (2006) New patients, new lessons, new thinking in inflammatory bowe disease: World Congress of Gastroenterology Symposium, Montreal, Canada. Colorectal Dis 8 Suppl 1(s1):1-2.

27. Gassanov N, Caglayan E, Semmo N, Massenkeil G, Er F (2014) Cirrhotic cardiomyopathy: a cardiologist's perspective. World J Gastroenterol 20: 15492-15498. [Crossref]

28. Chayanupatkul M, Liangpunsakul S (2014) Cirrhotic cardiomyopathy: review of pathophysiology and treatment. Hepatol Int 8: 308-315. [Crossref]

29. Møller S, Henriksen JH, Bendtsen F (2014) Extrahepatic complications to cirrhosis and portal hypertension: haemodynamic and homeostatic aspects. World J Gastroenterol 20: 15499-517. [Crossref]

30. Møller S, Bendtsen F (2015) Cirrhotic Multiorgan Syndrome. Dig Dis Sci 60: 3209 3225. [Crossref]

Copyright: (C2017 Lanzieri P. This is an open-access article distributed under the terms of the Creative Commons Attribution License, which permits unrestricted use, distribution, and reproduction in any medium, provided the original author and source are credited. 